\title{
MALIGNANT MELANOMA OF NOSE
}

\author{
I.N. Kundu', B. Haldar ${ }^{2}$, A. K. Saha ${ }^{3}$
}

\begin{abstract}
Malignant melanoma $(M M)$ is one of the uncommon malignancies of the nose. We present an unusually big proliferative like $M M$ in the vestibule of the nose.

Malignancy of nose constitutes less than $1 \%$ of all malignancies $(3 \%$ of head \& neck tumour). $M M$ however contributes only $2 \%$ of all malignant neoplasms of the nose (Moore \& Martin, 1955).
\end{abstract}

\section{CASE REPORT}

A 70 years old male patient attended ENT out patient department of NRS Medical College \& Hospitals Calcutta with a rapidly growing proliferative mass over the ala of the nose involving the right vestibule. It appeared initially as tiny warty lesion inside the right vestibule 6 months back which grew rapidly since last 2 months involving the ala of the same side and occluding the nostril also. He had no past history of radiation. He had no habit of smoking and taking alcohol. He had no pain or bleeding from the lesion. According to him, the growth was so ugly on his face that he came for treatment out of anxiety and RMO cum Clinical Tutor, 2PGT(MS), 3Associate cosmetic reason, as well.

Clinical Examination revealed a proliferative growth over the right ala of the nose involving the vestibule and occluding the nostril of the same side with a broad base. The size of the tumour was $4 \mathrm{~cm} \mathrm{x} 3 \mathrm{~cm}$ with an irregular uneven surface and variegated in appearance and feel with pigmented haemorrhagic black spots over th0e surface with some areas of ulceration (Fig. I \& II). It was firm to hard in consistency with restricted mobility with induration but without any tenderness. There was no palpable regional lymph node. Septum of the nose was not involved and

\footnotetext{
${ }^{1}$ RMO cum Clinical Tutor, ${ }^{2}$ PGT(MS), ${ }^{3}$ Associate Professor, Deptt. of E.N.T.,N R S Medical College, Calcutta.
} 


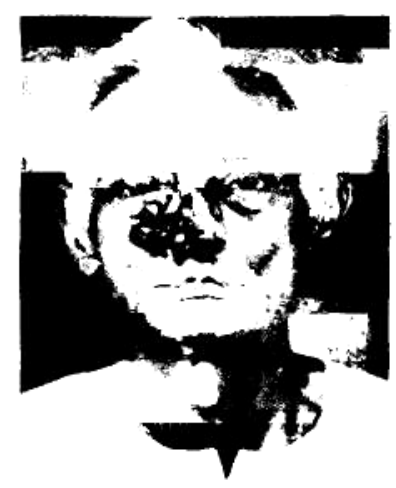

Fig. I : Pre-operative photograph of the patient from front

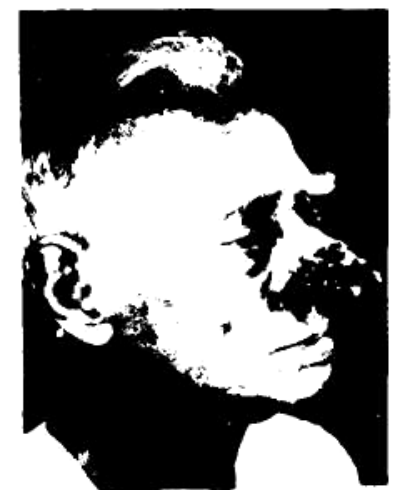

Fig. II : Pre-operative photograph of the patient from side

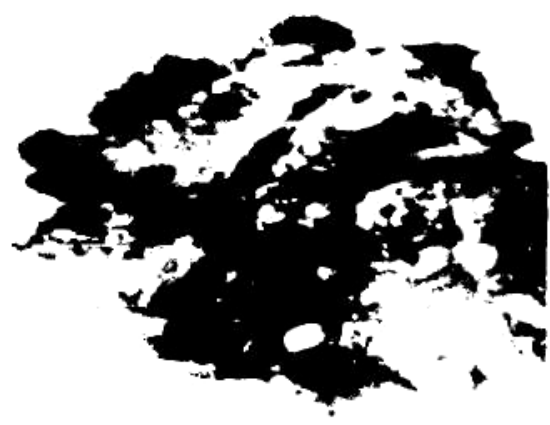

Fig. III : Specimen of excised tumour

other vestibule and nasal cavity were normal. Examination of the other parts of the nose, nasopharynx, throat and ear revealed no abnormality. Few scattered nevus were seen over the body.

The patient was prepared for surgery. Routine

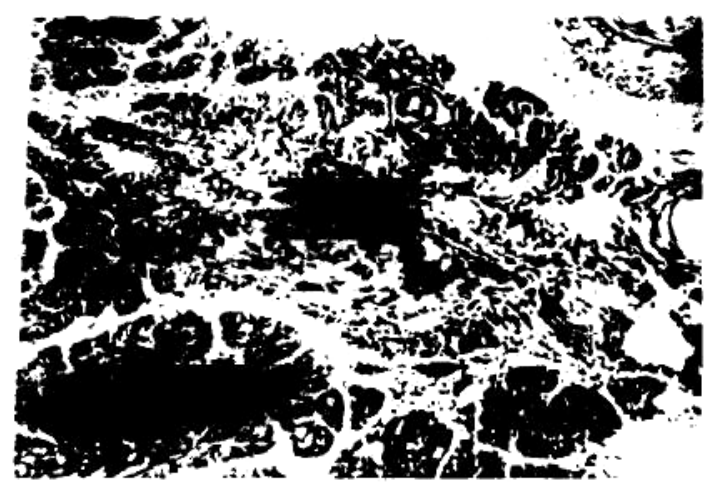

Fig. IV : Histopathological picture of the tumour

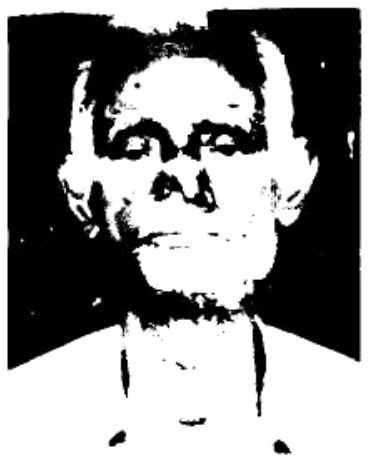

Fig. V : Post-operative photograph of the patient

haematological parameters including serum urea, creatinine, blood sugar and liver function test were within normal limits. X-ray of chest (PA view) and X-ray of PNS (OM view) were within normal limits. Pre-operative minute cutting biopsy of the swelling suggested the diagnosis of Malignant Melanoma. Complete wide excision of the mass (more than $2 \mathrm{~cm}$. healthy margin all around) was done under general anaesthesia. The operation was uneventful. The specimen (Fig. III) was sent for serial section histopathology study. Macroscopically, the excised mass was polypoid with some ulceration and pigmentation. Microscopically, it showed pleomorphism with melanocytes. The sarcoma-like, spindle-celled form was evident. Melanin was abundant. These features established the diagnosis of MM (Fig.IV). The stitches were removed on the $7^{\text {th }}$ day post-operative day. The patient was kept under watch with careful follow-up for local recurrence or appearance of node.

The patient however showed no recurrence of disease 
during post-operative follow-up period of 6 months at the time of writing this case report. The only problem recorded was the stenosis of right nasal cavity (Fig. V). We are meticulously monitoring the patient for any local recurrence as well as nodal and distant metastasis.

\section{DISCUSSION}

$\mathrm{MM}$ is comparatively rare in comparison with squamous carcinoma among the cancers that arise in the nasal region (Friedmann \& Osborn, 1982). Most of the tumours arise at the vestibule of the nose and very few in the nasal cavities and sinuses. Melanocytes in the nasal cavity can be found in the respiratory epithelium and nasal gland, (site of apparent predilection for MM) (Zak \& Lawson, 1974). Although the olfactory epithelium is rich in melanin pigment, no $\mathrm{MM}$ has been found to arise from this epithelium. Basal cell carcinoma or rodent ulcer involving the ala and vestibule of the nose is different but may give similar appearance. There is no sex difference pertinent to $\mathrm{MM}$ of the nose. The peak age is from fourth to six the decades of life. The biological course of the nasal MM is marked by early local recurrences and extension and frequent metastasis to lymph nodes and viscera e.g. lever (Gallagher, 1970). In our case however, no lymph node was palpable at the time of surgery.

Regarding treatment, excision of the MM with a healthy margin of $2 \mathrm{~cm}$ all around is widely accepted (Abide et al, 1984) where clinically no palpable regional lymph node is detected and in case of metastatic node, suprahyoid dissection is to be undertaken. Radiotherapy appears to have little effect on the majority of the MM (Harrison, 1976). It may play a role in the management of the inoperable recurrences (Freedman et al, 1973).

Mohs' surgery (Microscopically controlled surgical excision) is definitely indicated in treatment of large MM with ill-defined boarders in peri nasal \& nasal areas where recurrence rates are high (Mohs, 1977). It is the microscopically controlled dermatological surgery performed under local anesthesia in case of smaller size of growth. In bigger size MNs, general anesthesia is necessary. During surgery, the growth is removed layer by layer with each layer examined under microscope until no cancer cell can be found.

Regarding cryotherapy, it is favoured for very small lesions and the elderly debilitated patients (Bethesda, 1992). The carbon-dioxide laser is a cutting tool with the advantage of producing haemostasis of small vessels while cutting.
It has the advantage of keeping blood loss to a minimum in surgery of MM. Regarding chemotherapy, Dacarbazine may be used for palliative treatment only.

$\mathrm{MM}$ is the least common but most dangerous form of nasal cancer. MM usually begins as a darkly pigmented or irregular mole. Cytologically MM may be of different histological types. Clerk (1969) and Breslow (1970) have given the beautiful clinical and histological description of MM. MM often spreads to other parts of the body in its later stages of development. Early detection and treatment of $\mathrm{MM}$ is important in managing this potentially lifethreatening form of nasal cancer.

\section{ACKNOWLEDGEMENT}

Authors are thankful to the Superintendent of NRS Medical College \& Hospital, Calcutta for allowing to use the hospital records to publish the case.

\section{REFERENCES}

1. Moore, E.S. and Martin. H. (1955) : Melanoma of the upper respiratory tract and oral cavity. Cancer $8: 1167$.

2. Friedman, I. and Osborn, D.A. (1982): Pathology of granulomas and neoplasms of the nose and paranasal sinuses. Edinburgh : Churchill Livingstone, 162.

3. Zak, F.G. and Lawson, W. (1974): The presence of melanocytes in the nasal cavity. Ann. Otol. 83:515.

4. Gallagher, J.C. : Upper respiratory melanoma. Pathology and growth rate. Ann. Otol. Rhinol. Laryngol. 79:551.

5. Abide, J.M., Nahai, F. and Bennett, R.G. (1984): The meaning of Surgical margins. Plast. Reconstr. Surg. 73 : 492 - 496.

6. Harrison, D.F. (1976) : Malignant melanomata arising in the nasal mucous membrane. J. Laryngol. 90:993.

7. Freedman, H.M.. DeSanto, L.W., Devene, K.D. and Weiland, L.H. (1973) : Malignant melanoma of the nasal cavity and paranasal sinuses. Arch Otolaryngol. 97:322.

8. Mohs, F. E. (1977) : Chemosurgery for the microscopically controlled excision of cutaneous cancer. In : Epstein, E., Epstein, Jr., eds. Skin surgery, 4th ed. Springfield, IL : Charles C. Thomas : 526-541.

9. Bethesda, M.D. (1992): Physician's Data Query. Cancer of the skin. Nat'/ Cancer Inst, Jan 1992.

10. Clerk, W.H. (1969) : Am J. Path. 39 - 55.

11. Breslow. (1970) : Ann of Surgery. 172 - 902.

\section{Address for Communication :} DR. INDRANATH KUNDU

AE-147, Sector -I, Salt Lake City, Calcutta - 700064. 